\title{
Coherent forward scattering peak and multifractality
}

\author{
Maxime Martinez $\odot,{ }^{1}$ Gabriel Lemarié $\odot,{ }^{1,2,3}$ Bertrand Georgeot $\odot,{ }^{1}$ Christian Miniatura $\odot, 2,3,4,5,6$ and Olivier Giraud $\odot^{7}$ \\ ${ }^{1}$ Laboratoire de Physique Théorique, Université de Toulouse, CNRS, UPS, Toulouse, France \\ ${ }^{2}$ MajuLab, CNRS-UCA-SU-NUS-NTU International Joint Research Unit, Singapore \\ ${ }^{3}$ Centre for Quantum Technologies, National University of Singapore, 117543 Singapore, Singapore \\ ${ }^{4}$ Université Côte d'Azur, CNRS, INPHYNI, Nice, France \\ ${ }^{5}$ Department of Physics, National University of Singapore, 117542 Singapore, Singapore \\ ${ }^{6}$ School of Physical and Mathematical Sciences, Nanyang Technological University, 637371 Singapore, Singapore \\ ${ }^{7}$ Université Paris-Saclay, CNRS, LPTMS, 91405 Orsay, France
}

(Received 5 November 2020; revised 2 July 2021; accepted 28 July 2021; published 13 August 2021)

\begin{abstract}
It has recently been shown that interference effects in disordered systems give rise to two nontrivial structures: the coherent backscattering (CBS) peak, a well-known signature of interference effects in the presence of disorder, and the coherent forward scattering (CFS) peak, which emerges when Anderson localization sets in. We study here the CFS effect in the presence of quantum multifractality, a fundamental property of several systems, such as the Anderson model at the metal-insulator transition. We focus on Floquet systems, and find that the CFS peak shape and its peak height dynamics are generically controlled by the multifractal dimensions $D_{1}$ and $D_{2}$, and by the spectral form factor. We check our results using a one-dimensional Floquet system whose states have multifractal properties controlled by a single parameter. Our predictions are fully confirmed by numerical simulations and analytic perturbation expansions on this model. Our results, which we believe to be generic, provide an original and direct way to detect and characterize multifractality in experimental systems.
\end{abstract}

DOI: 10.1103/PhysRevResearch.3.L032044

Introduction. In the field of quantum transport, the coherent backscattering (CBS) effect is a well-known signature of interference effects that emerges at a time of the order of the elastic scattering time, and survives configuration average in time-reversal symmetric disordered systems [1-4]. It is visible as a peak in momentum space for spatially disordered systems and in position space for the type of Floquet systems that we consider in the present Letter [5]. Recently, it was discovered that, in the presence of Anderson localization, CBS was further accompanied by the emergence of a coherent forward scattering (CFS) peak (which actually arises even without time-reversal symmetry), leading to a twin-peak structure breaking ergodicity in the long-time limit [6].

The CFS peak is in fact a smoking gun of strong localization [5-12]. In particular, it was shown [11] that it could be used to monitor the metal-insulator Anderson transition, as it vanishes in the metallic phase and is fully developed in the localized regime. It was even suggested [11] that at the transition, where there exist nonergodic delocalized states with multifractal properties [13-15] (i.e., scale-invariant fluctuations characterized by a continuous set of fractal dimensions $D_{q}$ ) the CFS peak might embody these multifractal properties. In this Letter, we consider the particular case of

Published by the American Physical Society under the terms of the Creative Commons Attribution 4.0 International license. Further distribution of this work must maintain attribution to the author(s) and the published article's title, journal citation, and DOI.
Floquet systems where localization and/or multifractality occur in momentum space. This type of dynamical systems is extremely convenient for extensive numerical studies and has been already implemented in several experiments (see, e.g., Refs. $[16,17])$. For these kicked systems, we demonstrate that the height and shape of the CFS peak (a dynamical observable in position space) give direct and remarkable access to the multifractal dimensions $D_{1}$ and $D_{2}$ (a static property of the Floquet eigenstates in momentum space). Our general predictions are very well corroborated by numerical simulations and analytical perturbative expansions on the RuijsenaarsSchneider model [18], a dynamical system where all states have multifractal properties controlled by a single parameter. Since our results are based on a very general theoretical framework and derived using well-supported arguments, we believe that they should apply to any critical disordered system.

This work paves the way to a robust experimental study of quantum multifractality, that remains very hard to observe despite huge theoretical interest (see the pioneering experiments in a quantum setting [19,20] and in a classical setting [21]). Indeed, the CFS peak is a direct experimental observable that has recently been observed with cold atom experiments in the localized regime [22].

The Ruijsenaars-Schneider (RS) model. The RS model [18] is a variant of the kicked rotor [23,24], a paradigmatic model of quantum chaos which exhibits Anderson localization in momentum space. It is a one-dimensional (1D) Floquet system whose corresponding Hamiltonian reads $H=$ $p^{2} / 2-2 \pi a[x(\bmod 2 \pi)] \sum_{n=-\infty}^{\infty} \delta(t-n)$, featuring a periodically kicked sawtooth potential with strength $2 \pi a$. The 
difference with the kicked rotor comes from the spatial discontinuities of the sawtooth potential, inducing long-range hopping between momentum basis states, which breaks standard exponential localization [13,25-29]. Saliently, the RS model displays multifractal eigenstates [30-33]. The dynamics of such periodically kicked systems is captured by the Floquet operator $U$ over one period, whose eigenvectors $\left|\varphi_{\alpha}\right\rangle$ are associated with quasienergies $\omega_{\alpha} \in[-\pi, \pi$, so that $U^{t}\left|\varphi_{\alpha}\right\rangle=e^{i \omega_{\alpha} t}\left|\varphi_{\alpha}\right\rangle$. For the RS model, $U$ can be written explicitly in the momentum basis $|p\rangle$ (with integer $p, 0 \leqslant p \leqslant$ $N-1)$ as

$$
\left\langle p|U| p^{\prime}\right\rangle=U_{p p^{\prime}}=\frac{e^{i \phi_{p}}}{N} \frac{1-e^{2 \pi i a}}{1-e^{2 \pi i\left(p^{\prime}-p+a\right) / N}},
$$

where the dynamical phases $\phi_{p}$ can be taken as randomly distributed over $[0,2 \pi$ [. In the following, we will denote disorder average by bracketed terms $\langle(\cdots)\rangle$. This random matrix ensemble has been intensively studied in many branches of theoretical physics and mathematics [18,34-42]. In particular, it breaks time-reversal symmetry, so that the usual CBS effect is destroyed [5,22], and its spectrum displays intermediate statistics [34].

It is important to recall that the eigenstates of $\mathrm{RS}$ are multifractal in momentum space [31,32]. This is characterized by the anomalous scaling of the disorder-averaged moments of the wave functions, $\left\langle\sum_{p}\left|\varphi_{\alpha}(p)\right|^{2 q}\right\rangle \sim N^{-D_{q}(q-1)}$, where $\varphi_{\alpha}(p) \equiv\left\langle p \mid \varphi_{\alpha}\right\rangle$ and $D_{q}$ are the multifractal dimensions. In the RS model, the parameter $a$ controls the nature of the eigenstates [31]: When $a$ goes from 0 to 1 , the system goes from the regime of strong multifractality $\left(D_{q} \ll 1\right)$ to the regime of weak multifractality $\left(D_{q} \sim 1\right)$, making it an ideal test bed for our theory.

The CFS contrast. The RS model above is only an example of a more general class of systems which can be described by an evolution operator $U$ with Floquet states localized or multifractal in momentum space. For such systems, the CFS interference phenomenon takes place in position space [5]. We introduce the position basis $|x\rangle(x=2 \pi n / N, 0 \leqslant n \leqslant N-1)$ which is related to the momentum basis $|p\rangle$ defined above by the Fourier transform $\langle x \mid p\rangle=\exp (i p x) / \sqrt{N}$.

In the following, we thus consider the time evolution of the system starting from some initial state $\left|x_{0}\right\rangle$ in position space and analyze the disorder-averaged position distribution after $t$ iterations of the map $U$, namely $\left\langle\left|\left\langle x\left|U^{t}\right| x_{0}\right\rangle\right|^{2}\right\rangle$. After an initial transient regime, it features a peak around the initial value $x=x_{0}$, the CFS peak. To single out this interference effect resisting disorder average, we introduce the contrast $\Lambda\left(x, x_{0}, t\right)$ as the relative difference between the quantum probability distribution $\left\langle\left|\left\langle x\left|U^{t}\right| x_{0}\right\rangle\right|^{2}\right.$ and the classical, interference-free, long-time limit $1 / N$ :

$$
\Lambda\left(x, x_{0}, t\right)=\frac{\left\langle\left|\left\langle x\left|U^{t}\right| x_{0}\right\rangle\right|^{2}\right\rangle-1 / N}{1 / N} .
$$

The time behavior of the contrast is illustrated in Fig. 1 in the case of the RS model. A peak emerges at short times around $x=x_{0}$, its height oscillates (left projection in Fig. 1), and eventually stabilizes (right projection).

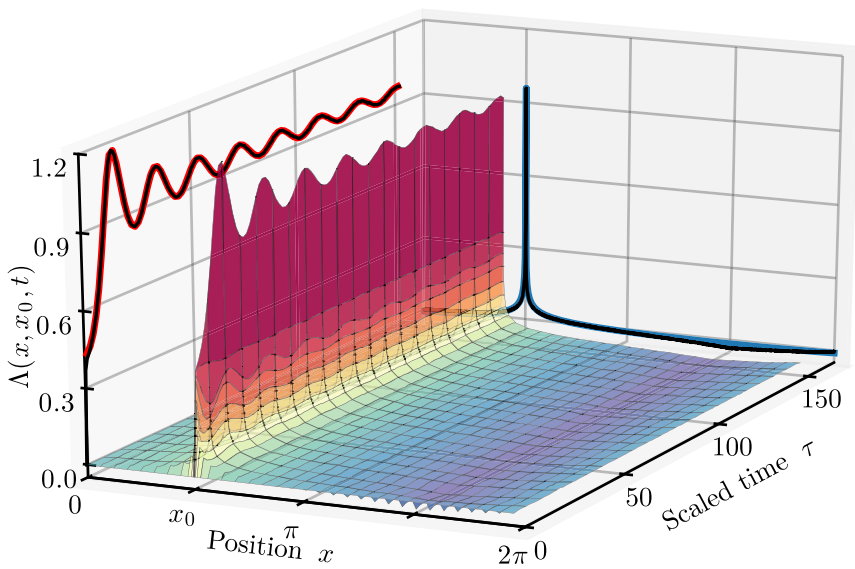

FIG. 1. Contrast of the CFS peak, Eq. (2), with scaled time $\tau=2 \pi t / N$, for the RS model with $N=4096$ and $a=0.322$. Left projection: Height of the CFS peak. The theoretical prediction (red solid line) [see text below Eq. (17)] matches perfectly the numerical data (black solid line). Right projection: Shape of the CFS peak at $\tau=150$. The black solid line represents the numerical data and the blue solid line is Eq. (9) rescaled (see text).

Expanding over eigenstates of $U$, the contrast (2) writes

$$
\Lambda\left(x, x_{0}, t\right)=N \sum_{\alpha \beta}\left\langle e^{i \omega_{\alpha \beta} t} \varphi_{\alpha}^{*}(x) \varphi_{\alpha}\left(x_{0}\right) \varphi_{\beta}^{*}\left(x_{0}\right) \varphi_{\beta}(x)\right\rangle-1,
$$

where $\varphi_{\alpha}(x) \equiv\left\langle x \mid \varphi_{\alpha}\right\rangle$ and $\omega_{\alpha \beta}=\left[\omega_{\alpha}-\omega_{\beta}\right](\bmod 2 \pi) \in$ $[-\pi, \pi]$. Note that in Eq. (3), $t$ can be considered a continuous variable: In the following we shall therefore resort to the usual Fourier transform rather than the discrete one.

At long times, only the diagonal part $\alpha=\beta$ in Eq. (3) survives, giving, for fixed system size $N$, the stationary limit

$$
\Lambda_{\infty}\left(x, x_{0}\right)=N \sum_{\alpha}\left\langle\left|\varphi_{\alpha}(x)\right|^{2}\left|\varphi_{\alpha}\left(x_{0}\right)\right|^{2}\right\rangle-1 .
$$

The time dependence of $\Lambda\left(x, x_{0}, t\right)$ is fully encapsulated in the off-diagonal terms $\alpha \neq \beta$. The function $F\left(x, x_{0}, t\right)=$ $\Lambda\left(x, x_{0}, t\right)-\Lambda_{\infty}\left(x, x_{0}\right)$ that governs the time dynamics of the contrast is given by the inverse Fourier transform of

$$
\hat{F}\left(x, x_{0}, \omega\right)=2 \pi N \sum_{\alpha \neq \beta}\left\langle\delta\left(\omega-\omega_{\alpha \beta}\right) \varphi_{\alpha}^{*}(x) \varphi_{\alpha}\left(x_{0}\right) \varphi_{\beta}^{*}\left(x_{0}\right) \varphi_{\beta}(x)\right\rangle .
$$

In what follows, we will first analyze the stationary (i.e., $t \rightarrow \infty)$ contrast $\Lambda_{\infty}\left(x, x_{0}\right)$ for finite $N$, and discuss its peak value at $x=x_{0}$ and its shape around $x_{0}$. Then, we will discuss the time dynamics of the peak at $x=x_{0}$, given by $F\left(x_{0}, x_{0}, t\right)$, and show that the limits of large times $t$ and large system sizes $N$ do not commute. These stationary distribution and time dynamics are illustrated in Fig. 1 for the RS model.

At this point, our strategy is to connect these dynamical quantities expressed in $x$ space to the known multifractal properties of the Floquet eigenstates in $p$ space. For this, we use the spatial Fourier transform and introduce the four-point correlator in momentum space:

$$
C_{\alpha \beta}\left(p_{1}, p_{1}^{\prime}, p_{2}, p_{2}^{\prime}\right)=\left\langle\varphi_{\alpha}\left(p_{1}\right) \varphi_{\alpha}^{*}\left(p_{1}^{\prime}\right) \varphi_{\beta}\left(p_{2}\right) \varphi_{\beta}^{*}\left(p_{2}^{\prime}\right)\right\rangle .
$$



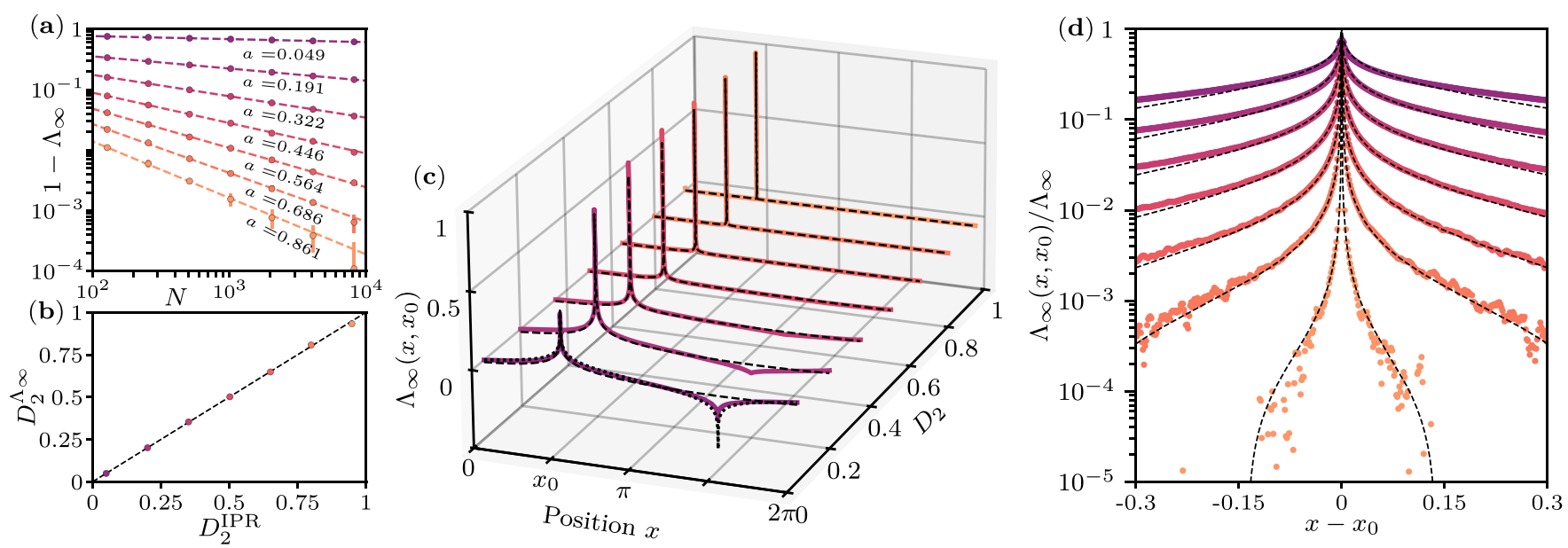

FIG. 2. Contrast of the CFS peak at infinite time. (a) Finite-size scaling of $\Lambda_{\infty}$ for different values of $a$. The dashed lines correspond to the nonlinear fit $1-\Lambda_{\infty}=\alpha N^{-D_{2}^{\Lambda}}$ with parameters $\alpha$ and $D_{2}^{\Lambda_{\infty}}$. (b) Comparison between the values of $D_{2}$ extracted from a finite-size scaling of $\Lambda_{\infty}$ in (a) and from the inverse participation ratio (IPR) defined by the right-hand side of Eq. (8). The two coincide very well. When not visible, error bars are smaller than the symbol size. (c) CFS peak contrast for the values of $a$ used in (a), with $N=4096$. Solid lines are numerical data, black dashed lines are Eq. (9) rescaled to minimize the difference with numerics on a spatial range $\Delta x=0.5$ around $x=x_{0}$ (excluding the point at $x_{0}$ ), and the black dotted line is Eq. (10). (d) Zoom-in of (c) around $x_{0}$ and smoothed over $\delta x=0.007$.

As is well known, the scaling properties of the correlator (6) encapsulate the multifractal dimensions [13]. Additionally, following the rationale behind random matrix theory (RMT), we assume that phases and norms of each wave function in the correlator $C_{\alpha \beta}$ are independent random variables, so that only terms where phase factors cancel survive the disorder average.

Stationary contrast and $D_{2}$. The stationary contrast $\Lambda_{\infty}\left(x, x_{0}\right)$ defined in Eq. (4) can be expanded in the momentum basis as $\frac{1}{N} \sum_{\alpha} \sum_{p_{1} p_{1}^{\prime} p_{2} p_{2}^{\prime}} C_{\alpha \alpha} e^{i\left[\left(p_{1}-p_{1}^{\prime}\right) x+\left(p_{2}-p_{2}^{\prime}\right) x_{0}\right]}-$ 1. Under the RMT assumption, the only nonvanishing terms left after disorder average are those with $p_{1}=p_{1}^{\prime}, p_{2}=$ $p_{2}^{\prime}$ and $p_{1}=p_{2}^{\prime}, p_{2}=p_{1}^{\prime}$. Taking care of double counting $\left(p_{1}=p_{2}^{\prime}=p_{2}=p_{1}^{\prime}\right)$ and making use of normalization $\left[\sum_{p}\left|\varphi_{\alpha}(p)\right|^{2}=1\right]$, we find

$$
\Lambda_{\infty}\left(x, x_{0}\right)=\frac{1}{N} \sum_{\alpha} \sum_{p_{1} \neq p_{2}}\left\langle\left|\varphi_{\alpha}\left(p_{1}\right)\right|^{2}\left|\varphi_{\alpha}\left(p_{2}\right)\right|^{2}\right\rangle e^{i\left(p_{1}-p_{2}\right)\left(x-x_{0}\right)} .
$$

The contrast at the tip of the peak, $\Lambda_{\infty} \equiv \Lambda_{\infty}\left(x_{0}, x_{0}\right)$, can be evaluated by rewriting Eq. (7) for $x=x_{0}$ as a sum over $p_{1}, p_{2}$ and subtracting its diagonal part. In contrast with systems with a mobility edge such as the Anderson model, all eigenvectors here have the same multifractal properties. The sum over $\alpha$, which is an average over eigenvectors, is then easily taken care of and we find

$$
\Lambda_{\infty}-1=-\sum_{p}\left\langle\left|\varphi_{\alpha}(p)\right|^{4}\right\rangle \propto N^{-D_{2}},
$$

where $\varphi_{\alpha}(p)$ is an arbitrary eigenvector. The right-hand side of (8) is then obtained using the well-known multifractal scaling of the inverse participation ratio (IPR) [13]. Thus, remarkably, the CFS contrast is directly related to the multifractal dimension $D_{2}$. The prediction Eq. (8) is very well verified in our model (see Fig. 2). The contrast around the peak can be obtained in the same manner from Eq. (7) by using the multifractal scaling of the correlation function, $\left\langle\left|\varphi_{\alpha}\left(p_{1}\right)\right|^{2}\left|\varphi_{\alpha}\left(p_{2}\right)\right|^{2}\right\rangle \sim\left|p_{1}-p_{2}\right|^{D_{2}-1} / N^{D_{2}+1} \quad[43,44]$, which yields

$$
\Lambda_{\infty}\left(x, x_{0}\right) \propto \frac{1}{N} \sum_{p=1}^{N-1} \cos \left[p\left(x_{0}-x\right)\right]\left(1-\frac{p}{N}\right)\left(\frac{p}{N}\right)^{D_{2}-1} .
$$

Here again, this general prediction directly links $\Lambda_{\infty}\left(x, x_{0}\right)$ to the multifractal dimension $D_{2}$. Note that Eq. (9) can actually be seen as a power-law decay $\sim\left|x-x_{0}\right|^{-D_{2}}$ for $x$ close to $x_{0}$ [see Supplemental Material (SM) [45]]. As shown in Fig. 2, Eq. (9) is also in very good agreement with numerical results for the RS model and reproduces quite well the spatial profile of the contrast in the region around $x=x_{0}$.

Remarkably, the behavior Eq. (8) can even be checked analytically in the RS model. Indeed, using a perturbation expansion at finite $N$ in the regime of strong multifractality $a \ll 1$, we get at first order in $a$ [46] the expressions $D_{2}=a$ and

$$
\begin{aligned}
\Lambda_{\infty}\left(x, x_{0}\right)= & 2 D_{2} \sum_{p=1}^{N-1} \frac{\pi\left(1-\frac{p}{N}\right)}{N \sin \frac{p \pi}{N}} \sin \left[p\left(x+\frac{\pi}{N}\right)\right] \\
& \times \sin \left[p\left(x_{0}+\frac{\pi}{N}\right)\right],
\end{aligned}
$$

which for $x=x_{0}$ leads to $\Lambda_{\infty} \sim D_{2} \log N$. Since $1-$ $\gamma N^{-D_{2}} \sim D_{2} \log N$ for $a \ll 1$, Eq. (8) is verified analytically at first order in $a$ for the RS model. Note that the dip at $x=-x_{0}$ in Fig. 2(c), which is an idiosyncrasy of our model, is well described by Eq. (10) (see Ref. [46] for details).

In the stationary limit $t \rightarrow \infty$ taken at finite system size $N$, the spatial profile of the CFS peak for a system with multifractal eigenstates is thus controlled by the multifractal dimension $D_{2}$. In particular, Eq. (8) shows that the peak height value $\Lambda_{\infty}=1$ that was found in Refs. [7,8,10,11] for disordered models and in Ref. [5] for the kicked rotor in the localized regime when $N \gg \xi \gg 1$, is reached here with an algebraic finite-size correction $N^{-D_{2}}$, a signature of multifractality. 
Time dynamics of the CFS peak height and $D_{1}$. We now aim at describing the temporal evolution of the CFS peak height $\Lambda\left(x_{0}, x_{0}, t\right)=\Lambda_{\infty}+F\left(x_{0}, x_{0}, t\right)$. Starting from Eq. (5) and assuming eigenvector and eigenvalue decorrelation under disorder average when $x=x_{0}$, we get

$$
\hat{F}\left(x_{0}, x_{0}, \omega\right)=\frac{2 \pi}{N} \hat{R}(\omega) \sum_{\alpha \neq \beta}\left\langle\delta\left(\omega-\omega_{\alpha \beta}\right)\right\rangle,
$$

where the correlator $\hat{R}(\omega)=N^{2}\left\langle\left|\varphi_{\alpha}\left(x_{0}\right)\right|^{2}\left|\varphi_{\beta}\left(x_{0}\right)\right|^{2}\right\rangle_{\omega_{\alpha \beta}=\omega}$ only involves eigenfunctions whose quasienergies are exactly separated by $\omega$ and does not depend on the labels $\alpha$ and $\beta$ because of disorder averaging. This implies that we can write the contrast as a convolution product,

$$
\Lambda\left(x_{0}, x_{0}, t\right)=\Lambda_{\infty}+\left[\left(K_{N}-1\right) \otimes R\right](t),
$$

where $R(t)$ is the inverse Fourier transform of $\hat{R}(\omega)$ and $K_{N}(t)=\left\langle\frac{1}{N}\left|\operatorname{tr} U^{t}\right|^{2}\right\rangle=1+\frac{1}{N}\left\langle\sum_{\alpha \neq \beta} e^{i \omega_{\alpha \beta} t}\right\rangle$ is the spectral form factor.

To compute $R(t)$ in Eq. (12), we follow the same steps as in the previous section: We expand the correlator $\hat{R}(\omega)=$ $\sum_{p_{1} p_{1}^{\prime} p_{2} p_{2}^{\prime}} C_{\alpha \beta}\left(p_{1}, p_{1}^{\prime}, p_{2}, p_{2}^{\prime}\right) e^{i\left[\left(p_{1}-p_{1}^{\prime}+p_{2}-p_{2}^{\prime}\right) x_{0}\right]}$ in momentum space, where $C_{\alpha \beta}$ in Eq. (6) is computed for eigenfunctions with $\omega_{\alpha \beta}=\omega$, and only keep terms surviving disorder average. We find $\hat{R}(\omega)=1-\sum_{p}\left\langle\left|\varphi_{\alpha}(p)\right|^{2}\left|\varphi_{\beta}(p)\right|^{2}\right\rangle_{\omega_{\alpha \beta}=\omega}$. Writing $\sum_{p}\left\langle\left|\varphi_{\alpha}(p)\right|^{2}\left|\varphi_{\beta}(p)\right|^{2}\right\rangle_{\omega_{\alpha \beta}=\omega}=\sum_{p}\left\langle\left|\varphi_{\alpha}(p)\right|^{4}\right\rangle \hat{C}(\omega)$, three regimes can be identified for multifractal wave functions $[13,43]$ :

$$
\hat{C}(\omega)=C_{0} \begin{cases}1, & \omega<\omega_{0}, \\ \left(\omega / \omega_{0}\right)^{D_{2}-1}, & \omega_{0} \leqslant \omega \leqslant \omega_{1}, \\ N^{D_{2}-1}\left(\omega / \omega_{1}\right)^{-2}, & \omega_{1} \leqslant \omega,\end{cases}
$$

where $\omega_{0}$ is proportional to the mean level spacing $2 \pi / N$, $\omega_{1} \propto N \omega_{0}$, and $C_{0}$ is some numerical factor. We checked numerically that such an $\omega$ dependence is well verified in the $\mathrm{RS}$ model with $\omega_{0}=2 \pi a / N$ and the caveat that only the last two regimes are visible (see Refs. $[45,46]$ ) since there are no eigenstates separated by $\omega<\omega_{0}$ for this model [34].

We now note that the form factor at large $N$ is well approximated by the continuum limit $K_{N}(t)=\delta(\tau)+K_{\text {reg }}(\tau)$ [47] where the underlying $N$ dependence only appears through the scaled time $\tau=2 \pi t / N$. For the RS model, $K_{\text {reg }}(\tau)$ can be obtained analytically [34] and reads

$$
K_{\mathrm{reg}}(\tau)=\frac{(1-a)^{2}(a \tau)^{2}}{a^{2}(1-\cos a \tau)^{2}+[a \sin a \tau+(1-a) a \tau]^{2}} .
$$

The sinusoidal terms in Eq. (14) come from the existence of a nonzero minimal level spacing in the RS model and are actually responsible for the temporal oscillations of the contrast. In the following, we will assume that this continuum limit holds for the form factor.

Let us now discuss the main result of this section. We rewrite Eq. (12) as

$$
\Lambda\left(x_{0}, x_{0}, \tau\right)-K_{\mathrm{reg}}(\tau)=\delta(\tau)+\left(\Lambda_{\infty}-1\right) f(\tau),
$$

where

$$
f(\tau)=1+C(\tau)+\left[\left(K_{\text {reg }}-1\right) \otimes C\right](\tau) .
$$

Here, $C(t)$ is the inverse Fourier transform of $\hat{C}(\omega)$. From Eq. (13), we see that $C(\tau)$ and $f(\tau)$ do not depend on $N$ for $\tau>\tau_{1} \propto \frac{1}{N} \rightarrow 0$. After a very short fixed time $t_{1}=N \tau_{1} / 2 \pi$ $(\sim 1 / a$ for the RS model), we thus get

$$
\Lambda\left(x_{0}, x_{0}, \tau\right)-K_{\text {reg }}(\tau) \propto N^{-D_{2}} .
$$

This scaling law can be seen as a generalization of Eq. (8) at any time, and is very well verified in our model [see Fig. 3(a)]. At $\tau \gg \tau_{0}=2 \pi /\left(N \omega_{0}\right)$, because of the plateau in Eq. (13), we can even get an explicit approximation for $f(\tau)$ as $f_{\star}(\tau)=1+C_{0}\left[K_{\text {reg }}(\tau)-1\right]$; this gives $\Lambda\left(x_{0}, x_{0}, \tau\right)-$ $\left(\Lambda_{\infty}-1\right) f_{\star}(\tau)=K_{\text {reg }}(\tau)$, as illustrated in Fig. 3(b), which is a further illustration that $\Lambda\left(x_{0}, x_{0}, \tau\right)$ goes to $K_{\text {reg }}(\tau)$ at large $N$.

Noticeably, Eq. (17) actually implies that in the thermodynamic limit $(N, t \rightarrow \infty$ at fixed $\tau)$ the CFS contrast $\Lambda\left(x_{0}, x_{0}, \tau\right)$ is simply given by $K_{\text {reg }}(\tau)$ (which is the same result as in the localized regime $[7,8,11]$, because multifractal finite-size effects vanish in this limit). In particular, at $\tau \rightarrow 0^{+}$, the CFS contrast converges to the level compressibility $\chi=K_{\text {reg }}\left(\tau \rightarrow 0^{+}\right)$when increasing the system size $N$ [see Fig. 3(c)]. We recover here, and actually demonstrate in a very general framework, the relation that was recently conjectured at the Anderson transition [11], with an infinite system size at any fixed time $t$ (implying $\tau \rightarrow 0^{+}$). The link between the level compressibility and the multifractal dimensions $D_{q}$ has a long and controversial history $[13,48]$. However, the simple identity $\chi=1-D_{1} / d$ proposed in Ref. [49], with $d$ the dimension of the system, was verified both analytically and numerically in various systems [31,32], in particular in the RS model. Assuming this identity, together with the above considerations, demonstrates that the CFS contrast at any fixed nonzero time (or equivalently for $\tau \rightarrow 0^{+}$) goes to $\chi$ for $N \rightarrow \infty$ and thus gives direct access to $D_{1}$, as numerically demonstrated in Fig. 3(d):

$$
\lim _{N \rightarrow \infty} \Lambda\left(x_{0}, x_{0}, t\right)=1-D_{1} .
$$

Conclusion. In this Letter, we have shown that the CFS peak, a distinctive signature of Anderson localization, is also a marker of quantum multifractality, a fundamental property of several systems, such as the Anderson model at the metal-insulator transition. Our results are obtained for Floquet systems, but we believe them to be generic for critical disordered systems. Our work represents another situation which highlights the importance of studying physical observables in the space reciprocal to the space where localization, or multifractality, takes place [50-52]. Our results show that the multifractal dimensions $D_{1}$ and $D_{2}$ can unambiguously be measured from different dynamical quantities related to the CFS contrast. More precisely (i) the stationary shape of the CFS peak gives access to $D_{2}$ via Eq. (9), (ii) the finite-size scaling of the CFS contrast at any time gives $D_{2}$, Eqs. (8) and (17), and (iii) the limiting value of the CFS contrast at small $\tau$ gives $D_{1}$, Eq. (18). Remarkably, the CFS contrast is actually a direct state-of-the-art experimental observable, as was recently observed in the localized regime with a kickedlike system, using standard time-of-flight techniques [22]. In such kicked-like systems, the system size $N$ can be precisely 

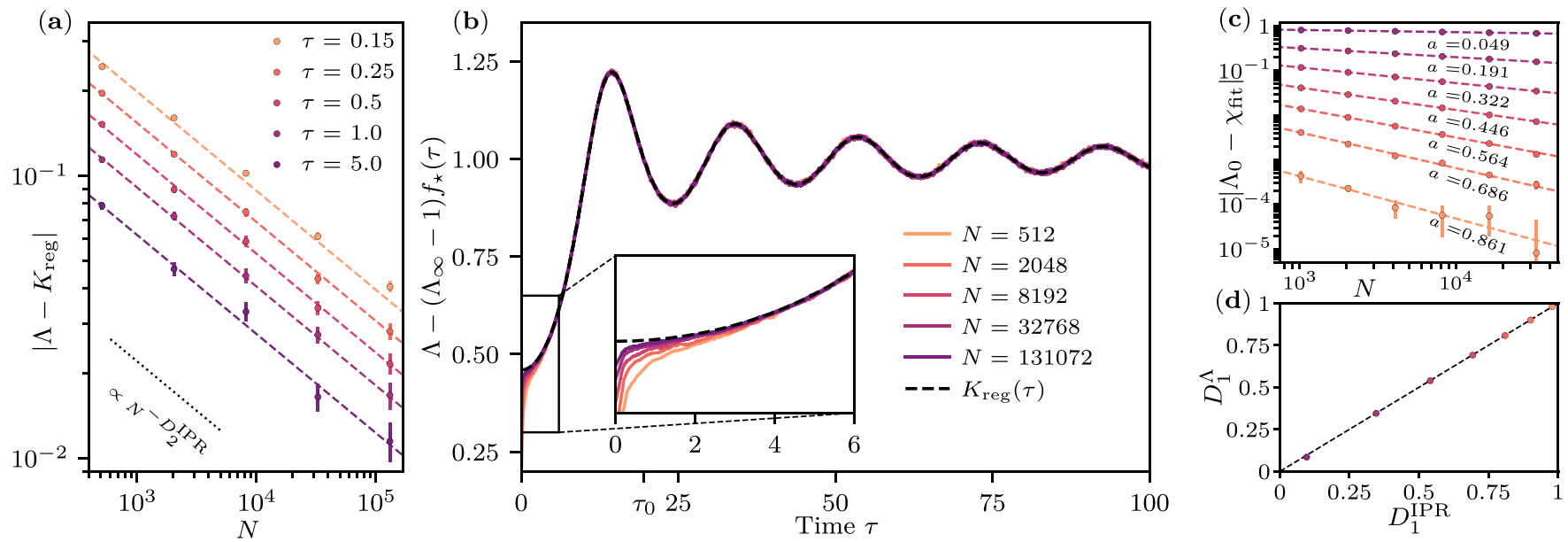

FIG. 3. Dynamics of the CFS peak at $x=x_{0}$. (a) Finite-size scaling of the difference between the numerically computed contrast and

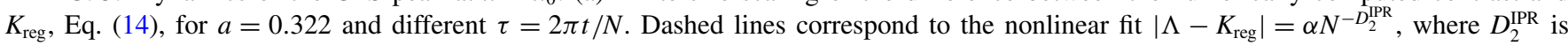
independently determined from a finite-size scaling of the IPR and $\alpha$ is the fitting parameter. (b) Dynamics of the contrast for different $N$. Solid lines are numerical data smoothed over $\Delta \tau=0.3 . C_{0}=0.55$ in $f_{\star}(\tau)$ (see text) is numerically extracted from $\hat{C}(\omega)$ (see SM [45]). $\Lambda_{\infty}$ for large $N$ is extrapolated from Fig. 2(a). The black dashed line is $K_{\text {reg }}$ given by Eq. (14). (c) Finite-size scaling of the difference between the contrast at $\tau=0.5$ and the level compressibility $\chi_{\mathrm{fit}}$ for different values of $a$. $\chi_{\mathrm{fit}}$ is extracted from a nonlinear fit $\Lambda=\chi_{\mathrm{fit}}+\alpha N^{-D_{2}^{\mathrm{IPR}}}$. (d) $D_{1}=1-\chi_{\text {fit }}$ vs $D_{1}$ obtained from a finite-size scaling of the wave-function moments. When not visible, error bars are smaller than the symbol size.

controlled, which should make it possible to reveal and monitor finite-size effects. Although the model (1) is difficult to implement directly with cold atoms because of the discontinuity of the potential [41,53], we think a suitably chosen temporal modulation could reproduce its main properties, as in Ref. [22]. In addition, it could also be implemented with photonic crystals [54-56]. Furthermore, we believe our results are generic enough to be relevant to other experimental systems where quantum multifractality is predicted to appear. As is well known, quantum simulation experiments are often plagued by dephasing mechanisms that destroy quantum coherence at long times (note that in Ref. [57] it was shown that wave-packet dynamics in momentum space only reveal multifractal properties at very long times). Our results suggest that this complication can be circumvented in our case since measurements can be done at short times. Our study thus paves the way to direct and robust measurements of multifractal properties of a quantum system that are notoriously hard to access by other means. Since multifractality is also known to appear in interacting systems [58-61], a possible extension of this work could be to study the fate of CFS in the presence of interactions and their impact on our results.

Acknowledgments. C.M. wishes to thank S. Cristofari for stimulating discussions and Laboratoire Collisions Agrégats Réactivité and Laboratoire de Physique Théorique (IRSAMC, Toulouse) for their kind hospitality. This study has been supported through the EUR Grant NanoX No. ANR-17-EURE-0009 in the framework of the "Programme des Investissements d'Avenir," and research funding Grants No. ANR-17-CE30-0024, No. ANR-18-CE30-0017, and No. ANR-19-CE30-0013. We thank Calcul en Midi-Pyrénées (CALMIP) for computational resources and assistance.
[1] E. Akkermans and G. Montambaux, Mesoscopic Physics of Electrons and Photons (Cambridge University Press, Cambridge, UK, 2011).

[2] N. Cherroret, T. Karpiuk, C. A. Müller, B. Grémaud, and C. Miniatura, Coherent backscattering of ultracold matter waves: Momentum space signatures, Phys. Rev. A 85, 011604(R) (2012).

[3] F. Jendrzejewski, K. Müller, J. Richard, A. Date, T. Plisson, P. Bouyer, A. Aspect, and V. Josse, Coherent Backscattering of Ultracold Atoms, Phys. Rev. Lett. 109, 195302 (2012).

[4] G. Labeyrie, T. Karpiuk, J.-F. Schaff, B. Grémaud, C. Miniatura, and D. Delande, Enhanced backscattering of a dilute Bose-Einstein condensate, Europhys. Lett. 100, 66001 (2012).

[5] G. Lemarié, C. A. Müller, D. Guéry-Odelin, and C. Miniatura, Coherent backscattering and forward-scattering peaks in the quantum kicked rotor, Phys. Rev. A 95, 043626 (2017).

[6] T. Karpiuk, N. Cherroret, K. L. Lee, B. Grémaud, C. A. Müller, and C. Miniatura, Coherent Forward Scattering Peak induced by Anderson Localization, Phys. Rev. Lett. 109, 190601 (2012).

[7] K. L. Lee, B. Grémaud, and C. Miniatura, Dynamics of localized waves in one-dimensional random potentials: Statistical theory of the coherent forward scattering peak, Phys. Rev. A 90, 043605 (2014).

[8] S. Ghosh, N. Cherroret, B. Grémaud, C. Miniatura, and D. Delande, Coherent forward scattering in two-dimensional disordered systems, Phys. Rev. A 90, 063602 (2014).

[9] T. Micklitz, C. A. Müller, and A. Altland, Strong Anderson Localization in Cold Atom Quantum Quenches, Phys. Rev. Lett. 112, 110602 (2014). 
[10] S. Ghosh, D. Delande, C. Miniatura, and N. Cherroret, Coherent Backscattering Reveals the Anderson Transition, Phys. Rev. Lett. 115, 200602 (2015).

[11] S. Ghosh, C. Miniatura, N. Cherroret, and D. Delande, Coherent forward scattering as a signature of Anderson metal-insulator transitions, Phys. Rev. A 95, 041602(R) (2017).

[12] M. Marinho and T. Micklitz, Spectral correlations in Anderson insulating wires, Phys. Rev. B 97, 041406(R) (2018).

[13] F. Evers and A. D. Mirlin, Anderson transitions, Rev. Mod. Phys. 80, 1355 (2008).

[14] Y. V. Fyodorov, A. Ossipov, and A. Rodriguez, The Anderson localization transition and eigenfunction multifractality in an ensemble of ultrametric random matrices, J. Stat. Mech. (2009) L12001.

[15] A. D. Mirlin and F. Evers, Multifractality and critical fluctuations at the Anderson transition, Phys. Rev. B 62, 7920 (2000).

[16] F. Moore, J. C. Robinson, C. Bharucha, P. E. Williams, and M. G. Raizen, Observation of Dynamical Localization in Atomic Momentum Transfer: A New Testing Ground for Quantum Chaos, Phys Rev. Lett. 73, 2974 (1994).

[17] J. Chabé, G. Lemarié, B. Grémaud, D. Delande, P. Szriftgiser, and J.-C. Garreau, Experimental Observation of the Anderson Metal-Insulator Transition with Atomic Matter Waves, Phys Rev. Lett. 101, 255702 (2008).

[18] S. N. M. Ruijsenaars and H. Schneider, A new class of integrable models and its relation to solitons, Ann. Phys. (NY) 170, 370 (1986).

[19] M. Morgenstern, J. Klijn, C. Meyer, and R. Wiesendanger, Real-Space Observation of Drift States in a Two-Dimensional Electron System at High Magnetic Fields, Phys. Rev. Lett. 90, 056804 (2003).

[20] A. Richardella, P. Roushan, S. Mack, B. Zhou, D. A. Huse, D. D. Awschalom, and A. Yazdani, Visualizing critical correlations near the metal-insulator transition in $\mathrm{Ga}_{1-x} \mathrm{Mn}_{x} \mathrm{As}$, Science 327, 665 (2010).

[21] S. Faez, A. Strybulevych, J. H. Page, A. Lagendijk, and B. A. van Tiggelen, Observation of Multifractality in Anderson Localization of Ultrasound, Phys. Rev. Lett. 103, 155703 (2009).

[22] C. Hainaut, I. Manai, J.-F. Clément, J.-C. Garreau, P. Szriftgiser, G. Lemarié, N. Cherroret, D. Delande, and R. Chicireanu, Controlling symmetry and localization with an artificial gauge field in a disordered quantum system, Nat. Commun. 9, 1382 (2018).

[23] B. V. Chirikov, A universal instability of many-dimensional oscillator systems, Phys. Rep. 52, 263 (1979).

[24] F. M. Izrailev, Simple models of quantum chaos: Spectrum and eigenfunctions, Phys. Rep. 196, 299 (1990).

[25] T. H. Seligman, J. J. M. Verbaarschot, and M. R. Zirnbauer, Spectral fluctuation properties of Hamiltonian systems: The transition region between order chaos, J. Phys. A: Math. Gen. 18, 2751 (1985).

[26] X. Deng, V. E. Kravtsov, G. V. Shlyapnikov, and L. Santos, Duality in Power-law Localization in Disordered OneDimensional Systems, Phys. Rev. Lett. 120, 110602 (2018).

[27] P. A. Nosov, I. M. Khaymovich, and V. E. Kravtsov, Correlation-induced localization, Phys. Rev. B 99, 104203 (2019).

[28] A. D. Mirlin, Y. V. Fyodorov, F.-M. Dittes, J. Quezada, and T. H. Seligman, Transition from localized to extended eigenstates in the ensemble of power-law random banded matrices, Phys. Rev. E 54, 3221 (1996).

[29] L. S. Levitov, Delocalization of Vibrational Modes caused by Electric Dipole Interaction, Phys. Rev. Lett. 64, 547 (1990).

[30] O. Giraud, J. Marklof, and S. O'Keefe, Intermediate statistics in quantum maps, J. Phys. A: Math. Gen. 28, L303 (2004).

[31] E. Bogomolny and O. Giraud, Perturbation approach to multifractal dimensions for certain critical random matrix ensembles, Phys. Rev. E 84, 036212 (2011).

[32] E. Bogomolny and O. Giraud, Multifractal dimensions for all moments for certain critical random-matrix ensembles in the strong multifractality regime, Phys. Rev. E 85, 046208 (2012).

[33] A. M. García-García and J. Wang, Anderson Transition in Quantum Chaos, Phys. Rev. Lett. 94, 244102 (2005).

[34] E. Bogomolny, O. Giraud, and C. Schmit, Integrable random matrix ensembles, Nonlinearity 24, 3179 (2011).

[35] E. Bogomolny, O. Giraud, and C. Schmit, Random Matrix Ensembles Associated with Lax Matrices, Phys. Rev. Lett. 103, 054103 (2009).

[36] J. Martin, O. Giraud, and B. Georgeot, Multifractality and intermediate statistics in quantum maps, Phys. Rev. E 77, 035201(R) (2008).

[37] Y. V. Fyodorov and O. Giraud, High values of disordergenerated multifractals and logarithmically correlated processes, Chaos, Solitons Fractals 74, 15 (2015).

[38] H. W. Braden and R. Sasaki, The Ruijsenaars-Schneider model, Prog. Theor. Phys. 97, 1003 (1997).

[39] E. Bogomolny and C. Schmit, Spectral Statistics of a Quantum Interval-Exchange Map, Phys. Rev. Lett. 93, 254102 (2004).

[40] E. B. Bogomolny, R. Dubertrand, and C. Schmit, Spectral statistics of a quantum interval-exchange map: The general case, Nonlinearity 22, 2101 (2009).

[41] R. Dubertrand, I. García-Mata, B. Georgeot, O. Giraud, G. Lemarié, and J. Martin, Two Scenarios for Quantum Multifractality Breakdown, Phys. Rev. Lett. 112, 234101 (2014)

[42] I. Garcia-Mata, J. Martin, O. Giraud, and B. Georgeot, Multifractality of quantum wave packets, Phys. Rev. E 86, 056215 (2012).

[43] Y. V. Fyodorov and A. D. Mirlin, Strong eigenfunction correlations near the Anderson-localization transition, Phys. Rev. B 55, R16001 (1997).

[44] E. Cuevas and V. E. Kravtsov, Two-eigenfunction correlation in a multifractal metal and insulator, Phys. Rev. B 76, 235119 (2007).

[45] See Supplemental Material at http://link.aps.org/supplemental/ 10.1103/PhysRevResearch.3.L032044 for details on the analytical derivation of Eq. (10) and extended numerical data, which includes Refs. [14,15,29].

[46] B. Georgeot, O. Giraud, G. Lemarié, M. Martinez, and C. Miniatura (unpublished).

[47] F. Haake, Quantum signatures of chaos, Quantum Coherence in Mesoscopic Systems (Springer, Boston, 1991), pp. 583-595.

[48] J. T. Chalker, V. E. Kravtsov, and I. V. Lerner, Spectral rigidity and eigenfunction correlations at the Anderson transition, JETP Lett. 64, 386 (1996).

[49] E. Bogomolny and O. Giraud, Eigenfunction Entropy and Spectral Compressibility for Critical Random Matrix Ensembles, Phys. Rev. Lett. 106, 044101 (2011). 
[50] V. V. Volchkov, M. Pasek, V. Denechaud, M. Mukhtar, A. Aspect, D. Delande, and V. Josse, Measurement of Spectral Functions of Ultracold Atoms in Disordered Potentials, Phys. Rev. Lett. 120, 060404 (2018).

[51] N. Cherroret, T. Karpiuk, B. Grémaud, and C. Miniatura, Thermalization of matter waves in speckle potentials, Phys. Rev. A 92, 063614 (2015).

[52] T. Scoquart, T. Wellens, D. Delande, and N. Cherroret, Quench dynamics of a weakly interacting disordered Bose gas in momentum space, Phys. Rev. Research 2, 033349 (2020).

[53] R. Dubertrand, I. García-Mata, B. Georgeot, O. Giraud, G. Lemarié, and J. Martin, Multifractality of quantum wave functions in the presence of perturbations, Phys. Rev. E 92, 032914 (2015).

[54] T. Schwartz, G. Bartal, S. Fishman, and M. Segev, Transport and Anderson localization in disordered two-dimensional photonic lattices, Nature (London) 446, 52 (2007).

[55] Y. Lahini, A. Avidan, F. Pozzi, M. Sorel, R. Morandotti, D. N. Christodoulides, and Y. Silberberg, Anderson Localization and
Nonlinearity in One-Dimensional Disordered Photonic Lattices, Phys. Rev. Lett. 100, 013906 (2008).

[56] L. Levi, Y. Krivolapov, S. Fishman, and M. Segev, Hypertransport of light and stochastic acceleration by evolving disorder, Nat. Phys. 8, 912 (2012).

[57] P. Akridas-Morel, N. Cherroret, and D. Delande, Multifractality of the kicked rotor at the critical point of the Anderson transition, Phys. Rev. A 100, 043612 (2019).

[58] M. Amini, V. E. Kravtsov, and M. Müller, Multifractality and quantum-to-classical crossover in the Coulomb anomaly at the Mott-Anderson metal-insulator transition, New J. Phys. 16, 015022 (2014).

[59] I. S. Burmistrov, I. V. Gornyi, and A. D. Mirlin, Multifractality at Anderson Transitions with Coulomb Interaction, Phys. Rev. Lett. 111, 066601 (2013).

[60] Y. Y. Atas and E. Bogomolny, Multifractality of eigenfunctions in spin chains, Phys. Rev. E 86, 021104 (2012).

[61] Y. Y. Atas and E. Bogomolny, Calculation of multi-fractal dimensions in spin chains, Philos. Trans. R. Soc., A 2007, 20120520 (2014). 